\title{
DISTRIBUIÇÃO DA GORDURA CORPORAL EM PACIENTES COM E SEM DOENÇAS CRÔNICAS: USO DA RELAÇÃO CINTURA-QUADRIL E DO ÍNDICE DE GORDURA DO BRAÇO
}

\section{BODY FAT DISTRIBUTION IN PATIENTS WITH AND WITHOUT CHRONIC-DEGENERATIVE DISEASES: USE OF THE WAIST TO HIP RELATIONSHIP AND ARM FAT INDEX}

\author{
Anderson Marliere NAVARRO' \\ Michele Sun STEDILLE' \\ Maria do Rosário Del Lama UNAMUNO' \\ Júlio Sérgio MARCHINI ${ }^{1}$
}

\begin{abstract}
RESUMO
O objetivo deste trabalho foi avaliar a distribuição de gordura corporal, visceral e não visceral, em pacientes internados, medida por meio da antropometria. Foram avaliados 157 pacientes internados, distribuídos em dois grupos: Grupo I (95 pacientes portadores de doenças crônicas) e Grupo II (62 pacientes portadores de hepatopatias, pneumopatias, nefropatias, neoplasias, úlceras, gastrites, e doenças hematológicas), denominados PTodos. Em cada paciente, foram obtidas as seguintes medidas antropométricas: peso, altura, prega cutânea tricipital e circunferências (cintura e quadril). Os maiores valores dos parâmetros relação cintura/quadril e índice de gordura do braço foram encontrados no Grupo I e estes valores foram significativamente superiores quando comparados ao Grupo II. Os resultados demonstraram que, no grupo de pacientes estudados, houve uma distribuição de gordura corporal diferenciada entre pacientes Grupo I e Grupo II. Estas relações confirmam que os pacientes portadores de doenças crônicas (Grupo I) apresentam valores de relação cintura/quadril e índice de gordura do braço que são discriminatórios em relação aos dos pacientes portadores de hepatopatias, pneumopatias, nefropatias, neoplasias, úlceras, gastrites e doenças hematológicas (Grupo II).
\end{abstract}

Termos de indexação: obesidade, antropometria, doenças cardiovasculares, doença crônica, índice de massa corporal, constituição corporal.

\begin{abstract}
The objective of this work was to evaluate the distribution of corporal, visceral and not visceral fat, in hospitalized patients, measured by means of the anthropometry. The study evaluated 157 hospitalized patients, distributed in two groups: Group I (95 patients with chronic-degenerative diseases) and Group II (62 patients with liver, lung and kidney diseases, neoplasms, ulcers, gastritis, and hematologic diseases). The following anthropometric measurements were obtained from each patient: weight, height, triceps skinfold thickness and circumferences (waist and hip). The largest values of the parameters waist to hip relationship and arm fat index were found in the Group I and these values were
\end{abstract}

\footnotetext{
${ }^{(1)}$ Divisão de Nutrição Clínica, Departamento de Clínica Médica, Laboratório de Espectrometria de Massa, Faculdade de Medicina de Ribeirão Preto, Universidade de São Paulo. Av. Bandeirantes, 3900, $1^{\circ}$ Andar, Anexo A, 14049-900, Ribeirão Preto, SP, Brasil. Correspondência para/Correspondence to: A.M. NAVARRO. E-mail: jsmarchi@fmrp.usp.br; andersonnavarro@yahoo.com
} 
significantly superior when compared to the Group II. The results demonstrated that, in the group of patients studied, there was a differentiated distribution of body fat between patients Group I and Group II. These relationships confirm that the patients with chronic-degenerative diseases (Group I) present values of waist to hip relationship and arm fat index that are differentiating in relation to the patients with liver, lung and kidney diseases, neoplasms, ulcers, gastritis and hematologic diseases (Group II).

Index terms: obesity, anthropometry, cardiovascular diseases; chronic-disease, body mass index, body constitution.

\section{INTRODUÇÃO}

Nos últimos 30 anos, paralelamente ao aumento em número e longevidade da população, a relação entre doença degenerativa e obesidade ficou evidenciada (Seidell et al., 1992; Azevedo et al., 1999; Ko et al., 1999). Assim sendo, entre as principais causas de mortalidade na população adulta, várias estão associadas à obesidade e hábitos alimentares inadequados (Bray, 1989). Por exemplo, insuficiência coronariana, acidente vascular cerebral, diabetes melito e hipertensão arterial, entre outras (Kissebah, 1996; Rexrode et al., 1998).

Ainda que o vínculo entre obesidade e doença aterosclerótica seja evidente, (Kissebah, 1996) demonstrou que existe um valor preditivo aumentado para doença cardiovascular e a presença de gordura visceral manifestada pela grande concentração de gordura, predominantemente na região abdominal (Segal et al., 1987; Seidell et al., 1987; Pouliot et al., 1994). Por essa razão, a distribuição de gordura corporal teria maior valor preditivo para a doença cardiovascular do que a relação entre peso corpóreo e altura (Kissebah, 1996; Rexrode et al., 1998).

Como característica masculina, aceita-se que existe um maior acúmulo de gordura visceral na região abdominal e nas mulheres tal acúmulo ocorre principalmente na região gluteofemoral (Martin \& Jensen, 1991). Considerando este aspecto, homem e mulher de mesma idade e com o mesmo índice de massa corpórea têm seu risco cardiovascular aumentado na proporção do aumento de gordura visceral abdominal (Pouliot et al., 1994). Para se avaliar a gordura visceral, rotineiramente se usa medida da circunferência da cintura dividida pela circunferência do quadril, como indicativo da adiposidade visceral (Martin \& Jensen, 1991; Cronk \& Roche, 1998; Valera \& Hernández, 1997). Paralelamente, para se avaliar o tecido adiposo não visceral, temos o índice de gordura do braço (Vannucchi et al., 1992), representativo da gordura não visceral.

Assim o objetivo deste estudo foi avaliar a distribuição de gordura corporal, visceral e não visceral, em pacientes portadores de doenças crônicas (como por exemplo pacientes diabéticos, hipertensos, coronariopatas) e portadores de outras doenças internados na Clínica Médica do Hospital das Clínicas da Faculdade de Medicina de Ribeirão Preto (HCRP) - Universidade de São Paulo. Tal avaliação foi feita através do estudo da relação Cintura/ quadril, representativa da gordura visceral e do índice de gordura do braço, representativa da não visceral.

\section{CASUÍSTICA E MÉTODOS}

Avaliou-se pacientes internados no Hospital das Clínicas da Faculdade de Medicina de Ribeirão Preto, de ambos os sexos, portadores de diferentes doenças, agrupados em dois grupos de estudo, segundo os diagnósticos definidos no prontuário médico. Participaram do estudo pacientes com idades superiores a 35 anos e com diagnóstico de diabetes, hipertensão, doenças coronarianas, infartados, obesos, dislipidêmicos (doenças crônicas) e pacientes com diagnóstico de hepatopatias, pneumopatias, nefropatias, neoplasias, úlceras, gastrites e doenças hematológicas. Os pacientes que apresentavam em sua evolução clínica sinais de edema e ascite foram excluídos do estudo.

Os pacientes foram agrupados de acordo com os diagnósticos em dois grupos de estudo: Grupo I (pacientes portadores de doenças crônicas) e Grupo II (pacientes portadores de hepatopatias, pneumopatias, nefropatias, neoplasias, úlceras, gastrites e doenças hematológicas).

Foi realizada, em cada um dos pacientes, avaliação antropométrica utilizando peso, altura, prega cutânea tricipital, comprimento do braço e circunferências (cintura e quadril). Os dados foram coletados durante um período de seis meses seguidos. Todas as medidas antropométricas foram feitas em cada paciente em um único momento e por um único observador.

\section{Relação Cintura/Quadril (RCQ)}

Essa relação é estabelecida por meio de uma divisão da circunferência da cintura (Cct) (realizada no ponto médio entre a última costela e a crista/ilíaca) e pela medida da Circunferência do Quadril (Cq) - (realizada ao nível do trocânter maior do fêmur) medida por fita métrica inextensível. Resumindo temos $\mathrm{RCQ}=\mathrm{Cct} / \mathrm{Cq}$.

\section{Prega Cutânea}

A pregas cutâneas do tricipital foram medidas pinçando-se o tecido gorduroso subcutâneo entre o polegar 
e o indicador, afastada do músculo e, em seguida, medida por meio de um paquímetro Lange Skinfold Caliper, com pressão uniforme de $10 \mathrm{~g} / \mathrm{mm}^{2}, 1 \mathrm{~cm}$ acima do ponto pinçado, considerando-se a média de três medidas sucessivas. As medidas das pregas cutâneas foram padronizadas no lado não dominante de cada paciente (Durnin \& Womersly, 1974; Vannucchi et al., 1992).

\section{Índice de Gordura do Braço (IGB)}

Foi calculado através do quociente da medida da prega tricipital (Ptr) $(\mathrm{mm})$ e a Distância Olécrano-acromial (Doa) $\left(\mathrm{dm}^{2}\right)$ medida por fita métrica inextensível (Vannucchi et al., 1992) no braço não dominante. Resumindo temos $\mathrm{IGB}=\operatorname{Ptr} / \mathrm{Doa}^{2}\left(\mathrm{~mm} / \mathrm{dm}^{2}\right)$.

\section{Peso e Altura}

Os pacientes internados foram pesados pela manhã, após o desjejum, descalços e com o mínimo de vestimentas, em balança Filizola do tipo Plataforma, com precisão de $0,1 \mathrm{~kg}$. A altura foi medida usando-se uma haste metálica vertical com graduação de 0,5 cm, fixada à parede. O peso assim obtido (peso atual) foi usado no cálculo do Índice de Massa Corporal (IMC), mediante a sua fórmula: $\mathrm{IMC}=\mathrm{P} / \mathrm{A}^{2}$, onde $\mathrm{P}=$ peso atual (kg) e $A=$ altura (m) (Bray, 1989; Vannucchi, et al., 1996).

\section{Análise Estatística}

Os dados foram representados por meio da média e desvio-padrão. Em seguida, foram comparadas estatisticamente as possíveis diferenças $(p<0,05)$ entre os Grupos I e II por meio de análise de variância. Também procurou-se verificar a correlação existente entre diferentes pares de dados.

\section{RESULTADOS}

Foram avaliados 157 pacientes; 95 se encontravam no Grupo I (49 do sexo feminino e 46 masculino) e 62 no Grupo II (25 do sexo feminino e 37 masculino) (Tabela 1).

Ao comparar os diferentes grupos para RCQ, IGB e IMC foram encontrados, respectivamente, Grupo I (sexo feminino 0,94 $\pm 0,08 ; 2,06 \pm 0,67 \mathrm{~mm} / \mathrm{dm}^{2}$; e $27 \pm 5,00 \mathrm{~kg} / \mathrm{m}^{2}$ e sexo masculino $1,02 \pm 0,06$; $1,00 \mathrm{~mm} / \mathrm{dm}^{2} \pm 0,37$; e $27 \pm 4,00 \mathrm{~kg} / \mathrm{m}^{2}$ ) Grupo II (sexo feminino $0,87 \pm 0,07 ; 1,62 \pm 0,85 \mathrm{~mm} / \mathrm{dm}^{2}$; e $21 \pm 5 ; 00 \mathrm{~kg} / \mathrm{m}^{2}$ e sexo masculino $0,96 \pm 0,10 ; 0,66$ $\mathrm{mm} / \mathrm{dm}^{2} \pm 0,27$; e $\left.22 \pm 5,00 \mathrm{~kg} / \mathrm{m}^{2}\right)$. Quando se comparou os Grupos I e II, sexo masculino e feminino, todos estes dados foram estatisticamente diferentes $(p<0,05)$ para o IMC onde não foram encontradas diferenças entre os sexos para IMC. Desta forma o Grupo I (diagnósticos de doenças crônicas) comportam-se de maneira bastante diferenciada do Grupo II (diagnósticos de hepatopatias, pneumopatias, nefropatias, neoplasias, úlceras, gastrites, e doenças hematológicas). Resumindo, os pacientes do Grupo I apresentaram valores superiores de RCQ, IGB e IMC, em relação aos pacientes do grupo II. Lembrando que todos os pacientes do Grupo II não apresentavam sinais clínicos que caracterizavam ascite e/ou edema.

Tabela 1. Medidas antropométricas (média e desvio-padrão) para o sexo feminino, masculino e grupo de doenças.

\begin{tabular}{|c|c|c|c|c|c|c|c|c|c|c|}
\hline Grupos de estudo & $\mathrm{n}$ & Idade (anos) & Peso (kg) & Altura $(\mathrm{cm})$ & $\operatorname{IMC}\left(\mathrm{kg} / \mathrm{m}^{2}\right)$ & IGB $\left(\mathrm{mm} / \mathrm{dm}^{2}\right)$ & PCT $(\mathrm{mm})$ & Cct $(\mathrm{cm})$ & $\mathrm{Cq}(\mathrm{m})$ & RCQ \\
\hline \multicolumn{11}{|l|}{ Feminino } \\
\hline Grupo I & 49 & $61 \pm 14^{a}$ & $66 \pm 13^{a}$ & $155 \pm 7$ & $27 \pm 5^{a}$ & $2,06 \pm 0,67^{\mathrm{a}}$ & $23 \pm 8^{a}$ & $96 \pm 13^{a}$ & $102 \pm 12^{\mathrm{a}}$ & $0,94 \pm 0,08^{a}$ \\
\hline Grupo II & 25 & $46 \pm 19^{b}$ & $54 \pm 15^{b}$ & $159 \pm 7$ & $21 \pm 5^{b}$ & $1,62 \pm 0,85^{b}$ & $18 \pm 10^{b}$ & $81 \pm 11^{b}$ & $92 \pm 12^{b}$ & $0,87 \pm 0,08^{b}$ \\
\hline \multicolumn{11}{|l|}{ Masculino } \\
\hline Grupo I & 46 & $56 \pm 12^{a}$ & $77 \pm 14^{a}$ & $169 \pm 7$ & $27 \pm 4^{a}$ & $1,00 \pm 0,37^{\mathrm{a}}$ & $14 \pm 5^{a}$ & $100 \pm 12^{a}$ & $98 \pm 8^{\mathrm{a}}$ & $1,02 \pm 0,06^{\mathrm{a}}$ \\
\hline Grupo II & 37 & $51 \pm 17^{b}$ & $61 \pm 14^{b}$ & $168 \pm 8$ & $22 \pm 5^{b}$ & $0,66 \pm 0,27^{b}$ & $9 \pm 4^{b}$ & $86 \pm 4^{b}$ & $89 \pm 8^{b}$ & $0,96 \pm 0,10^{b}$ \\
\hline
\end{tabular}

Grupo I = pacientes com diagnóstico de doenças crônicas.

Grupo II = pacientes com dignóstico de hepatopatias, pneumopatias, nefropatias, neoplasias, úlceras, gastrites e doenças hematológicas.

$\mathrm{N}=$ número da amostra.

IMC = Índice de Massa Corporal.

IGB = Índice de Gordura do Braço.

PCT = Prega Cutânea Tricipital.

$\mathrm{Cct}=$ Circunferência da Cintura.

$\mathrm{Cq}=$ Circunferência do Quadril.

$a b=$ Letras Diferentes em uma Mesma Coluna $p<0,05$. 


\section{DISCUSSÃO}

Ashwell et al. (1985) avaliando a distribuição de gordura corporal por meio de tomografia e medidas de circunferência, encontraram correlação significativa com a RCQ e gordura intra-abdominal. As mulheres com uma distribuição centralizada de gordura (circunferência da cintura maior que a circunferência do quadril: chamadas de "maçãs") tendem a ter uma maior proporção da gordura intra-abdominal que mulheres com uma distribuição gorda periférica (circunferência da cintura menor que a do quadril: chamadas de "peras"). Nesta situação foi demonstrado que correlação entre a circunferência da cintura e gordura intra-abadominal é maior que a circunferência do quadril e a gordura intra-abdominal, em um mesmo grupo de pessoas. Assim as complicações da obesidade que são associadas com valores altos de RCQ podem se relacionar especificamente à quantidade de gordura intra-abdominal. Em geral, os homens podem ser considerados de alto risco de doenças crônicas se a RCQ। for maior que 0,95 ; as mulheres, se for maior que 0,80 (Pouliot et al., 1994; Valera \& Hernández, 1997; Molarius et al., 1999).

Os nossos resultados, mesmo considerando que a população estudada é intra-hospitalar, confirmam que a distribuição da gordura corporal (adiposidade), medida por meio da RCQ para o sexo feminino Grupo I e II $(0,94 \pm 0,08$ e $0,87 \pm 0,07)$ e sexo masculino $(1,02 \pm 0,06$ e $0,96 \pm 0,10)$, indicativo de uma maior concentração de gordura intra-abdominal, podendo constituir um grupo de risco para doenças crônicas (Bouchard et al., 1993; Mauriège et al., 1993; Rexrode et al., 1998).

Por outro lado, o IMC está diretamente correlacionado a massa corporal total, gordura corporal total e risco de doenças crônicas $(r>0,90)$ (Seidell et al., 1992; Huang et al., 1999). Neste estudo a RCQ (Grupo I) correlacionou positivamente com IMC $(r=0,25 \mathrm{p}<0,05)$. Em particular, a correlação entre o IMC e a circunferência da cintura e do quadril foram de respectivamente $r=0,79$ $(p<0,05)$ e $r=0,80(p<0,05)$. Estes achados estão de acordo com outros trabalhos (Seidell et al., 1992; Hodge et al., 1993; Molarius et al., 1999).

O IGB é uma medida utilizada para avaliar a reserva de gordura corporal, em especial, a gordura periférica não visceral. Esta medida tem se mostrado útil quando o peso e/ou a altura não podem ser obtidas, impossibilitando o cálculo do IMC; e como um outro dado para se avaliar a reserva adiposa (não visceral). Pacientes com valores acima de 2,98 $\pm 0,67$ e 1,93 \pm 0,75, respectivamente, para o sexo feminino e masculino são classificados portadores de excesso de gordura corporal (obesos) (Vannucchi et al., 1992). Confirmando este dado na literatura Jordão Jr (1994) comparando tomografia computadorizada com medidas antropométricas em diferentes grupos de pacientes, encontrou que o IGB correlaciona-se positivamente com a área gorda do braço medida por tomografia computadorizada.
O estudo da distribuição da gordura não visceral subcutânea para o Grupo I evidenciou um excesso de gordura e o mesmo não pode ser visto para o Grupo II, apesar de existir neste grupo, um número pequeno de pacientes com excesso de peso.

Neste estudo o IMC correlacionou-se positivamente com IGB em ambos os grupos de estudo sendo esta correlação mais forte no Grupo II. No entanto, é conveniente salientar que o IMC não distingui entre gordura visceral e periférica (Must et al., 1991). Esta distinção pode ser facilmente realizada pela análise dos valores de RCQ e IGB, pois a RCQ é uma medida direta da distribuição de gordura na região da cintura e o IGB considera o depósito periférico de gordura. Confirmando esta afirmação encontrou-se no presente estudo uma correlação negativa entre IGB e RCQ $(r=-0,41 p<0,05)$ para o Grupo I e para o Grupo II não houve correlação. Outra hipótese importante é que dados utilizados para avaliar depósito de gordura como o IGB, representativos da gordura não visceral, poderiam se comportar como indicativos de risco (Vannucchi et al., 1992). No entanto esta hipótese ainda precisa ser demonstrada em trabalhos futuros.

\section{CONCLUSÃO}

Os resultados encontrados em um grupo de pacientes hospitalizados confirmam, as evidências populacionais de que existe uma distribuição de gordura corporal diferenciada entre pacientes portadores de doenças crônicas e outras doenças. As medidas de Cct e $\mathrm{Cq}$, sua relação RCQ e IGB mostraram ser importantes para avaliar a distribuição de gordura corporal nos pacientes hospitalizados.

\section{REFERÊNCIAS BIBLIOGRÁFICAS}

ASHWELL, M., COLE, T.J., DIXON, A.K. Obesidade: nova perspicácia na classificação antropometrica de distribuição gorda mostrada por tomografia computadorizada. British Medical Journal, London, v.290, n.6483, p.1692-1694, 1985.

AZEVEDO, A., RAMOS, E., Von HAFE, P., BARROS, H. Upper-body adiposity and risk of myocardial infarction. Journal of Cardiovascular Risk, Philadelphia, v.6, n.5, p.321-325, 1999.

BOUCHARD, C., DESPRES, J.P., MAURIÈGE, P. Genetic and nongenetic determinants of regional fat distribution. Endocrine Reviews, Bethesda, v.14, n.1, p.72-93, 1993.

BRAY, G.A. Classification and evaluation of the obesities. Medical Clinics North America, Philadelphia, v.73, n.1, p.161-184, 1989.

CRONK, C.E., ROCHE, A.F. Race and soc-specific reference data for tricips and subscapular skinfolds and weight/stature. American Journal of Clinical Nutrition, Bethesda, v.35, n.2, p.347-354, 1982.

DURNIN, J.V.G.A., WOMERSLEY, J. Body fat assessed from total body density and its estimation from skinfold thickness: 
measurenents on 481 men and women aged from 16 to 72 years. British Journal Nutrition, Wallingford, v.32, n.1, p.77-97, 1974.

HODGE, A.M., DOWSE, G.K., ZIMMET, P.Z. Association of body mass index and waist-hip circumference ratio with cardiovascular disease risk factors in Micronesian Nauruans. International Journal of Obesity, Basingstoke, v.17, n.7, p.399407, 1993.

HUANG, Z., WILLETT, W.C., COLDITZ, G.A., HUNTER, D.J., MANSON, J.E., ROSNER, B., SPEIZER, F.E., HANKINSON, S.E. Waist circumference, waist: hip ratio, and risk of breast cancer in the nurses'Health Study. American Journal of Epidemiology, Baltimore, v.150, n.12, p.1316-1324, 1999.

JORDÃO JR. A. Uso da tomografia computadorizada na avaliação nutricional: comparação entre dados antropométricos, bioquímicos e biofísicos. Araraquara, 1994. 52p. Dissertação (Mestrado) - Faculdade de Ciências Farmacêuticas, UNESP, 1994.

KISSEBAH, A.H. Intra-abdominal fat: is it a major factor in developing diabetes and coronary artery disease. Diabetes Research and Clinical Practice, Clare, v.30, p.S25-S30, 1996.

KO, G.T.C., CHAN, J.C.N., COCKRAM, C.S., WOO, J. Prediction of hypertension, diabetes, dyslipidaemia or albuminuria using simple anthropometric indexes in Hong Kong Chinese. International Journal of Obesity, Basingstoke, v.23, n.11, p.1136-1142, 1999.

MARTIN, M.L., JENSEN, M.D. Effects of body fat distribution on regional lipolysis in obesity. Journal of Clinical Investigation, New York, v.88, n.2, p.609-613, 1991.

MAURIÀGE, P., DESPRES, J.P., MOORJANI, S., PRUD'HOMME, D., LAMARCHE, B., BOUCHARD, C., NADEAU, A., TREMBLAY, A., LUPIEN, P.J. Abdominal and femoral adipose tissue lipolysis and cardiovascular disease risk factors in men. European Journal of Clinical Investigation, Oxford, v.23, n.11, p.729-740, 1993.

MOLARIUS, A., SEIDELL, J.C., SANS, S., TUOMILEHTO, J., KUULASMAA, K. Waist and hip circumferences, and waist-hip ratio in 19 populations of the WHO MONICA project. International Journal of Obesity, Basingstoke, v.23, n.2, p.116125,1999

MUST, A., DALLAL, G.E., DIETZ, W.H. Reference data for obesity: $85^{\text {th }}$ and $95^{\text {th }}$ percentiles of body mass index $\left(\mathrm{wt} / \mathrm{ht}^{2}\right)$ and tricepsskinfold thickness. American Journal of Clinical Nutrition, Bethesda, v.53, n.5, p.839-846, 1991.

POULIOT, M.C., DESPRES, J.P., LEMIEUX, S., MOORJANI, S., BOUCHARD, C., TREMBLAY, A., NADEAU, A., LUPIEN, P.J. Waist circumference and abdominal sagittal diameter: best simple anthropometric indexes of abdominal visceral adipose tissue accumulation and related cardiovascular risk in men and women. American Journal Cardiology, New York, v.73, n.7, p.460-468, 1994.

REXRODE, K.M., CAREY, V.J., HENNEKENS, C.H., WALTERS, E.E., COLDITZ, G.A., STAMPFER, M.J., WILLETT, W.C., MANSON, J.A.E. Abdominal adiposity and coronary heart disease in women. Journal of the American Medical Association, Chicago, v.280, n.21, p.1843-1848, 1998.

SEGAL. K.R., DUNAIF, A., GUTIN, B., ALBU, J., NYMAN, A., PISUNYER, F.X. Body composition, not body weight, is related to cardiovascular disease risk factors and Sex hormones levels in men. Journal of Clinical Investigation, New York, v.80, n.4, p.1050-1055, 1987.

SEIDELL, J.C., CIGOLINI, M., CHARZEWSKA, J., ELLSINGER, B.M., DESLYPERE, J. P., CRUZ, A. Fat distribution in European men: a comparison of anthropometric measurements in relation to cardiovascular risk factors. International Journal of Obesity, Basingstoke, v.16, n.1, p.17-22, 1992.

SEIDELL, J.A., OOSTERLEE, A., THIJSSEN, M.A., BUREMA, J., DEURENBERG, P., HAUTVAST, J.G. Assessment of intra-abdominal and subcutaneous abdominal fat: relation between anthropometry and computed tomography. American Journal of Clinical Nutrition, Bethesda, v.45, n.1, p.7-13, 1987.

VALERA, Y.H., HERNÁNDEZ, H.R.A. Relación del índice cintura/ cadera con la masa y el porcentaje de grasa corporal. Archivos Latinoamericanos de Nutrición, Guatemala, v.47, n.4, p.315-322, 1997.

VANNUCCHI, H., CUNHA, D.F., DUTRA DE OLIVEIRA, J E., MARCHINI, J.S. Arm fat index as an alternative parameter in the assessment of nutritional status of hospitalized patients. Journal of Nutritional Medicine, London, v.3, p.31-34, 1992.

VANNUCCHI, H., UNAMUNO, M.R.L., MARCHINI, J.S. Avaliação do estado nutricional. Medicina, Ribeirão Preto, v.29, p.5-18, 1996.

Recebido para publicação em 28 de outubro de 1999 e aceito em 16 de fevereiro de 2000. 